University of Nebraska - Lincoln

DigitalCommons@University of Nebraska - Lincoln

US Department of Energy Publications

U.S. Department of Energy

2006

Photoionization of Xenon Clusters

John D. Bozek

Lawrence Berkeley National Laboratory,.JDBozek@lbl.gov

Bruce S. Rude

Lawrence Berkeley National Laboratory, Bruce_Rude@lbl.gov

A. L. D. Kilcoyne

Lawrence Berkeley National Laboratory, ALKilcoyne@lbl.gov

Follow this and additional works at: https://digitalcommons.unl.edu/usdoepub

Part of the Bioresource and Agricultural Engineering Commons

Bozek, John D.; Rude, Bruce S.; and Kilcoyne, A. L. D., "Photoionization of Xenon Clusters" (2006). US Department of Energy Publications. 316.

https://digitalcommons.unl.edu/usdoepub/316

This Article is brought to you for free and open access by the U.S. Department of Energy at DigitalCommons@University of Nebraska - Lincoln. It has been accepted for inclusion in US Department of Energy Publications by an authorized administrator of DigitalCommons@University of Nebraska - Lincoln. 


\title{
Photoionization of Xenon Clusters
}

\author{
John D. Bozek ${ }^{*}$, Bruce S. Rude*, and A.L. David Kilcoyne* \\ *Advanced Light Source, Lawrence Berkeley National Laboratory, Berkeley, CA 94720 USA
}

\begin{abstract}
High resolution photoelectron spectra of xenon clusters of about 100 atoms, measured with photon energies of 20 to $60 \mathrm{eV}$ are reported. Peaks due to the clusters are observed at lower binding energies than the corresponding atomic lines and exhibit different behaviors. The peak associated with the Xe $5 p_{1 / 2}$ state at higher binding energy is relatively unchanged with photon energy whereas the Xe $5 p_{3 / 2}$ peak changes its shape with increasing photon energy. The change is attributed to differences in the escape depth of the electrons at different kinetic energies and the bulk and surface components of the cluster.
\end{abstract}

Keywords: photoelectron spectroscopy, clusters, photoionization, xenon, partial cross sections PACS: 33.60.-q, 36.40.-c, 32.70.Jz

\section{INTRODUCTION}

Photoionization studies of clusters of atoms and/or molecules offer unique insight into the evolution of the electronic structure bulk solids from their constituent atomic/molecular building blocks. Amongst the various types of clusters, such as metallic, semiconductor, ionic, etc., rare gas clusters are the most weakly bound, held together by van der Waals forces. Being only weakly bound, clusters of rare gas atoms are difficult to study experimentally since they are easily dissociated. Mass spectroscopic measurements of rare gas clusters, for example, usually results in neutral and ionic evaporation and dissociation of the cluster upon ionization [1].

Photoelectron spectroscopy provides a suitable means to study rare gas clusters owing to the short interaction time of the photoelectron with the residual cluster ion, usually fsecs. The first rare gas clusters to be studied by photoelectron spectroscopy were dimers of Xe [2] followed by dimers of $\mathrm{Ar}$ and $\mathrm{Kr}$ [3]. Several of the six photoelectron bands expected from molecular orbital theory were observed for each of the rare gas dimers and used to optimize the potential energy surfaces of the ions. More recently, very high resolution PFI-ZEKE photoelectron spectroscopy measurements have been used to construct potential energy surfaces for $\mathrm{Ar}_{2}^{+}, \mathrm{Kr}_{2}{ }^{+}$, and $\mathrm{Xe}_{2}{ }^{+}$with unprecedented accuracy $[4,5]$. Valence level photoelectron spectra of larger clusters of rare gas atoms have also been reported previously [6,7], although the spectra are dominated by broad unresolved "bands" rather than vibrationally resolved peaks. Inner-valence and inner-shell levels of rare gas clusters have become accessible for photoelectron spectroscopy with the advent of $3^{\text {rd }}$ generation synchrotron light sources, and several examples have been reported recently [8].

Inner-shell and inner-valence photoelectron spectra of rare gas clusters are relatively simple, exhibiting peaks shifted to lower binding energies relative to the 
corresponding atomic lines. The cluster inner-shell photoelectron peaks are of about the same width as the corresponding atomic lines and can usually be resolved into two peaks due to contributions from atoms on the surface of the cluster and from atoms in the interior or bulk of the cluster [8]. The outer valence photoelectron spectra of rare gas clusters also exhibit peaks shifted to lower binding energies relative to the corresponding atomic levels. In contrast to the inner-shell, however, the outer valence photoelectron peaks are much broader than either the atomic lines or cluster innershell photoelectron lines and exhibit different widths for the two spin-orbit split components $[6,7]$.

To further explore the unique behavior of the valence levels of rare gas clusters, we have examined the photon energy dependent behavior of the photoelectron spectra of large clusters of Xe. In addition to probing the photoionization dynamics of the cluster at different photon energies, changes in the excitation energy will result in different kinetic energies of the photoelectrons, which will be sensitive to changes in escape depth for electrons leaving the bulk of the cluster.

\section{EXPERIMENTAL}

Clusters of Xe with an average size $<\mathrm{N}>\sim 100$ atoms were produced from pure xenon using a continuous skimmed supersonic expansion gas jet. The skimmer region is fitted with two $10001 \mathrm{~s}^{-1}$ turbo molecular pumps and maintained at pressures of $\leq 10^{-3}$ Torr when the jet is operating. The aperture or nozzle of the source can be cooled with liquid nitrogen and a resistive electric heater is used to maintain the desired temperature. For the results reported here, a $200 \mu \mathrm{m}$ aperture was used and maintained at a temperature of $170 \pm 1 \mathrm{~K}$. A $1 \mathrm{~mm}$ diameter conical skimmer separates the nozzle and surrounding vacuum from the interaction region and photoelectron spectrometer. Pressures of $\leq 10^{-5}$ Torr were maintained in this region throughout the experiment.

Condensation of clusters from rare gases in expansions is well understood and the resulting cluster size is related to the condensation parameter, $\Gamma^{*}$, which can in turn be parameterized by the stagnation pressure, $p_{0}$, nozzle diameter, $d$, nozzle temperature, $t_{0}$, and gas specific constant, $k$, according to [9]:

$$
\mathrm{G}^{*}=k \times p_{0} \times d^{0.85} \times T_{0}^{2.2875}
$$

Using the values given above along with a gas constant, $k$, for xenon of 5554 [9], an average cluster size $<\mathrm{N}>$ of 100 atoms results.

Photoelectron spectra were measured with photons from Beamline 10.0.1 at the Advanced Light Source using a Scienta SES-200 electron spectrometer set at the magic angle [10]. The beamline was operated at $7 \mathrm{meV}$ resolution for photon energies below $35 \mathrm{eV}$, at $15 \mathrm{meV}$ for $35 \mathrm{eV} \leq \mathrm{hv} \leq 50 \mathrm{eV}$ and at $20 \mathrm{meV}$ at higher photon energies. A pass energy of $5 \mathrm{eV}$ was used on the spectrometer with differing slit sizes to approximately match the electron spectrometer resolution with the photon bandwidth. 


\section{RESULTS AND DISCUSSION}

A photoelectron spectrum of the Xe $5 p$ valence region, including both the atomic lines and the bands from the clusters measured with a total experimental resolution of 8 $\mathrm{meV}$ is shown in Fig. 1. The spectrum has been calibrated to the binding energy of the $\mathrm{Xe} 5 p_{3 / 2}$ line at $12.1298 \mathrm{eV}$ [11]. The spectrum in Fig. 1 is similar to others published previously $[7,8]$ exhibiting sharp lines for the spin-orbit split atom $5 \mathrm{p}$ lines, and much broader lines shifted to lower binding energies from each of the atomic peaks. The relative intensity of the cluster peaks is smaller than in recently published spectra due to the smaller cluster size in the present study, 100 atoms versus 1000 atoms, and the greatly enhanced resolution over the previous results [8].

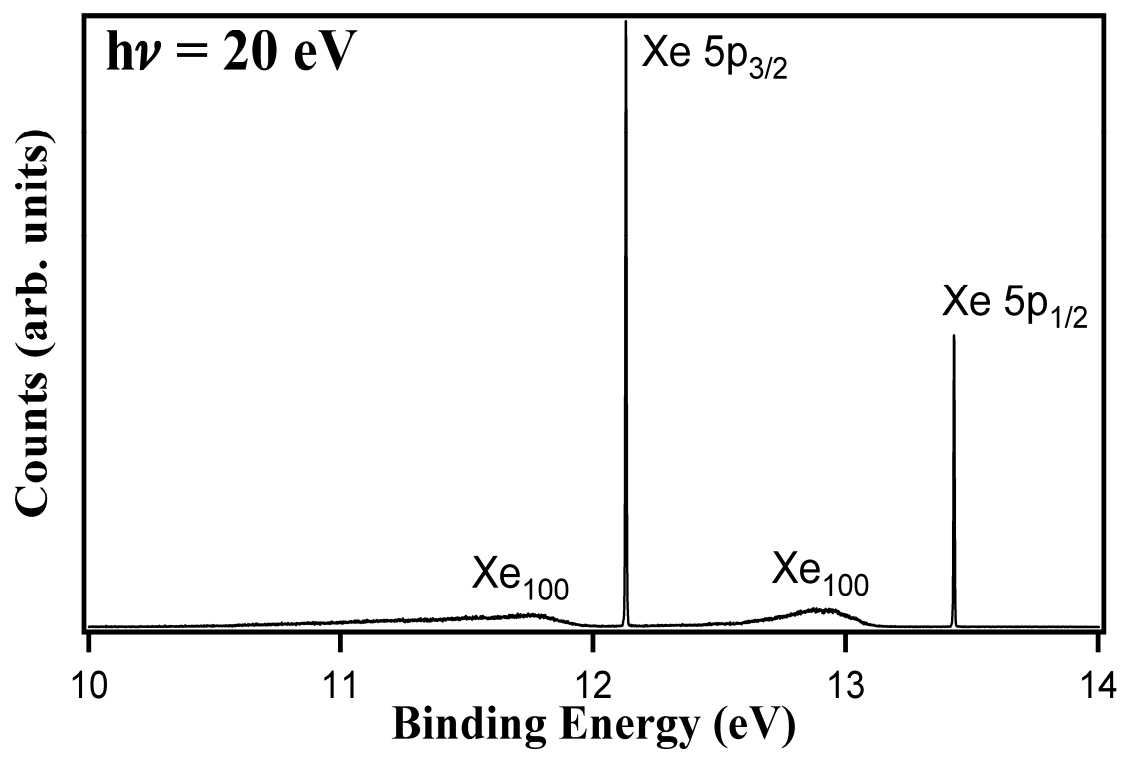

FIGURE 1. Photoelectron spectrum of Xe and clusters with average size of 100 atoms produced in supersonic jet expansion measured with a photon energy of $20 \mathrm{eV}$. The experimental resolution, as determined from the width of the $5 p_{3 / 2}$ line, is $8 \mathrm{meV}$

An enhanced view of the cluster peaks measured at the same photon energy but with a somewhat lower experimental resolution of $18 \mathrm{meV}$ is shown in Fig. 2. The two cluster peaks below the $\mathrm{Xe} 5 p_{3 / 2}$ and $\mathrm{Xe} 5 p_{1 / 2}$ lines are very different in shape, intensity, and energy separation from the corresponding atomic lines. The spectrum in Fig. 2 also exhibits considerably more structure than has been reported previously for outer valence photoelectron spectra of Xe clusters.

The difference in the shapes of the two cluster peaks is dramatic, with the peak closer to the Xe $5 p_{1 / 2}$ line being much narrower and almost Gaussian in shape with a full width at half maximum of about $300 \mathrm{meV}$ while the $\mathrm{Xe} 5 p_{3 / 2}$ cluster lines has a trigonal shape with a width at half height of about $750 \mathrm{meV}$. This disparity in the line shapes has been noted previously and attributed to either a $\mathrm{Xe}_{3}{ }^{+}$chromophore with strongly bound states associated with the ${ }^{2} \mathrm{P}_{3 / 2}$ ionization limit but only weakly bound 
or even unbound states for the ${ }^{2} \mathrm{P}_{1 / 2}$ ionization limit [7], or to the band structure of the condensed Xe cluster particle [8]. These results do not conclusively support either interpretation. Differences between the intensities of the two cluster peaks are somewhat deceptive, since the peak areas are much closer to expected statistical ratio of $2: 1$ due to the additional breadth of the $5 p_{3 / 2}$ cluster line.

Structure is apparent in the cluster peaks at binding energies of 12.96, 12.54 and $12.36 \mathrm{eV}$ for the $5 p_{1 / 2}$ line and $11.76,11.56,11.27$ and $10.9 \mathrm{eV}$ for the $5 p_{3 / 2}$ line. It is possible that given the relatively small size of the clusters studied here that a significant percentage of xenon dimer is present in the beam and may give rise to the narrower peaks. Peaks due to dimer were seen in low pressure spectra measured previously, that were quickly overwhelmed by signal from larger clusters as the size of the clusters was increased [7]. The positions of these bands are not in good agreement with previously reported $\mathrm{Xe}_{2}{ }^{+}$ionization energies, however [2,5]. In particular, the lack of intensity at $13.31 \mathrm{eV}$, where the $\mathrm{C}^{2} \Pi_{(1 / 2) \mathrm{u}}$ state was observed with the highest binding energy in previous photoelectron measurements [2] performed with He I radiation, contradicts the assignment of this structure to dimer contributions.

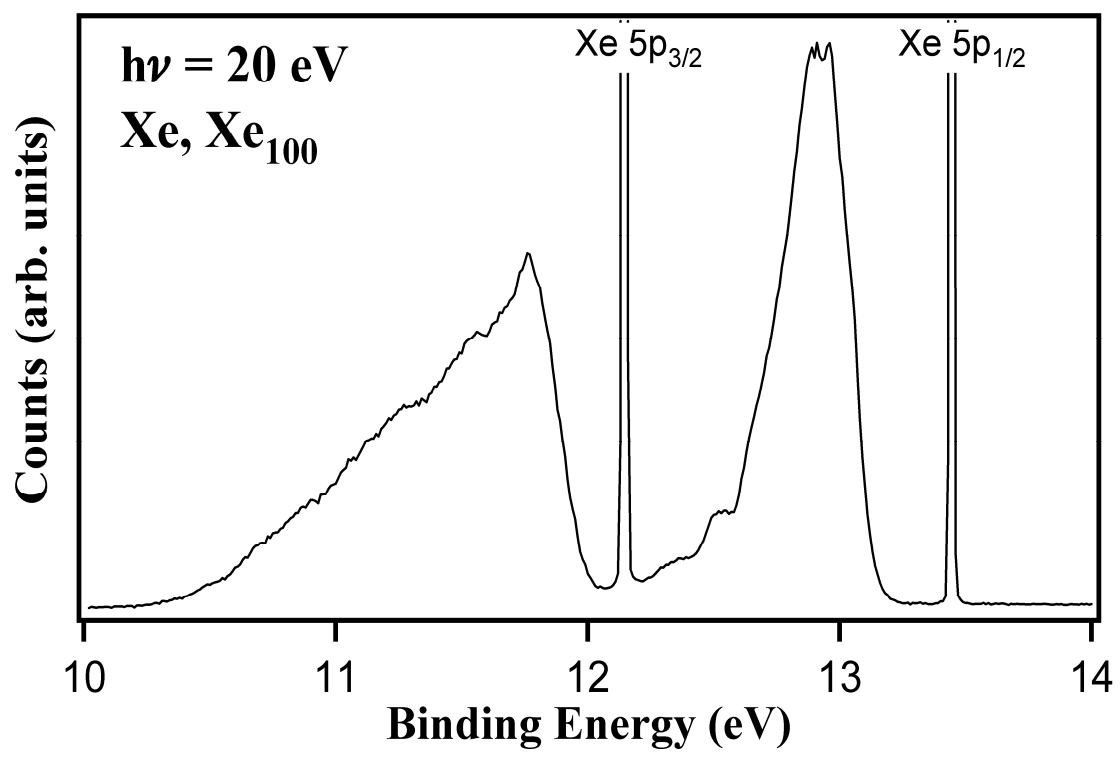

FIGURE 2. Photoelectron spectrum of Xe and clusters measured with a photon energy of $20 \mathrm{eV}$ vertically zoomed to enhance the intensity of the cluster peaks. The experimental resolution, as determined from the width of the $5 p_{3 / 2}$ line, is $18 \mathrm{meV}$

Xe $5 \mathrm{p}$ photoelectron spectra of the clusters were measured at additional photon energies, and the results are presented in Fig. 3. The higher photon energy spectra have somewhat poorer statistical quality, but several differences from the spectrum measured at $20 \mathrm{eV}$ photon energy emerge. Firstly, the Xe $5 p_{1 / 2}$ cluster peak is almost identical in shape and position across the sequence of energies, except for the $60 \mathrm{eV}$ spectrum, where the cluster peak is shifted to higher kinetic energy. This shift is due to a change in the stagnation pressure for this spectrum (due to the sample bottle 
running out) resulting in smaller clusters being produced in the beam. The average cluster size fell quickly from 100 to 80 atoms during the acquisition of this one spectrum. While the main cluster peak shifts to higher kinetic energy, the small peak apparent in several of the spectra at $12.64 \mathrm{eV}$ binding energy does not move. The other small peaks at 12.54 and $12.36 \mathrm{eV}$ appear to persist in the spectra at higher photon energies, although it is difficult to unambiguously identify them due to the noisier data.

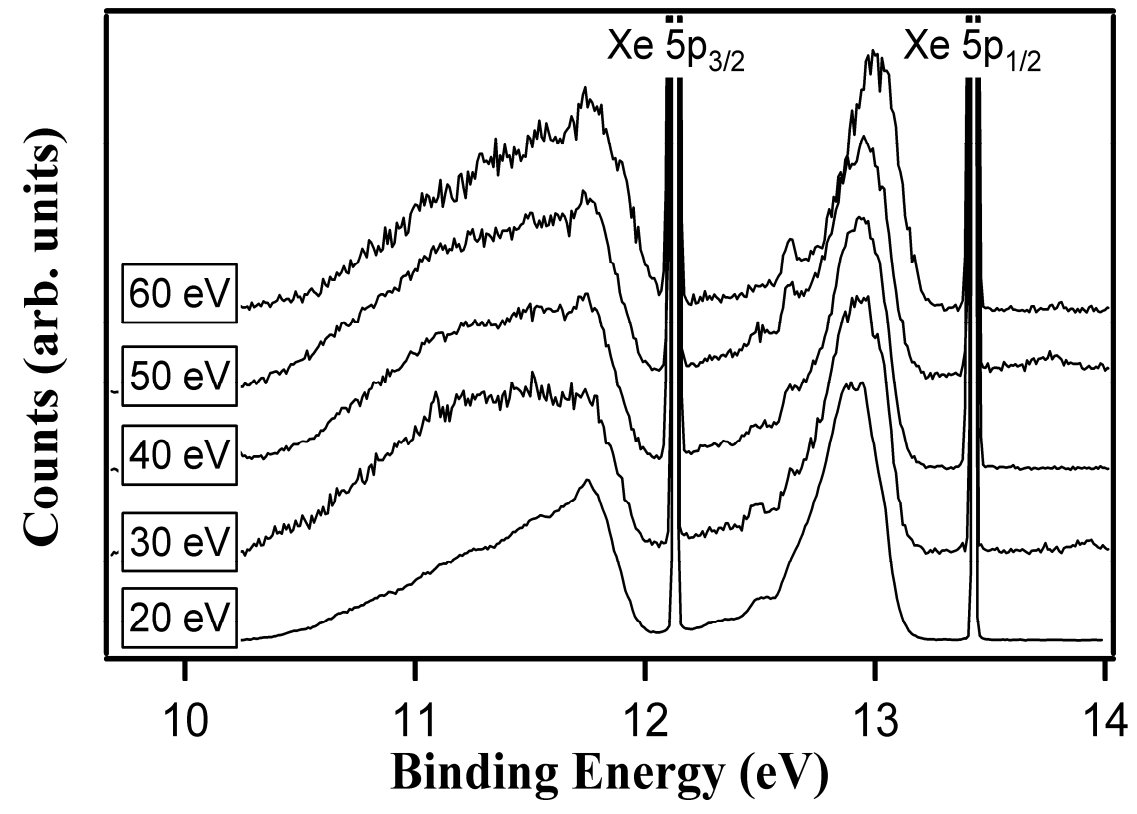

FIGURE 3. Photoelectron spectra of $\mathrm{Xe} 5 \mathrm{p}$ region measured at several different photon energies as indicated on the figure. The intensities were arbitrarily scaled to give the Xe $5 p_{1 / 2}$ cluster lines the same intensities in all of the spectra.

The $\mathrm{Xe} 5 p_{3 / 2}$ cluster peak changes dramatically at higher photon energies, exhibiting enhanced intensity at binding energies below the peak maximum of 11.76 $\mathrm{eV}$. The peak shape is similar for the spectra measured at 30,40 and $50 \mathrm{eV}$ although at $60 \mathrm{eV}$ the increased intensity begins to moderate and the shape is returning to that exhibited by the $20 \mathrm{eV}$ spectrum. The maximum of the peak at $11.76 \mathrm{eV}$ is remarkably constant, even for the $60 \mathrm{eV}$ spectrum where the peak broadens to higher binding energy rather than shifting. The origin of the additional intensity on the low binding energy side of the peak could simply be due to reduced escape depth for electrons emerging from the bulk of the cluster at these kinetic energies. The bulk/surface sensitivity ratio of $\mathrm{Xe} 4 \mathrm{~d}$ electrons for 300 atom and 1000 atoms clusters was found to exhibit a distinct minimum for kinetic energies between 20 and $40 \mathrm{eV}$ with a sharp maximum at lower kinetic energies and a slow increase at kinetic energies above 40 $\mathrm{eV}$ [12]. The present results are consistent with this model if the increased signal between 10.5 and $11.5 \mathrm{eV}$ binding energy is due to signal from the surface of the cluster while the underlying signal represented by the shape of the peak in the 
spectrum measured at $20 \mathrm{eV}$ photon energy is due primarily to atoms from the bulk of the cluster. Surprisingly, the Xe $5 p_{1 / 2}$ does not exhibit similar behavior, however.

\section{CONCLUSIONS}

High resolution outer valence photoelectron spectra of small xenon clusters of about 100 atoms have been measured using photon energies between 20 and $60 \mathrm{eV}$.

Consistent with previous results, the cluster spectra exhibit two broad bands shifted to lower binding energies than the corresponding atomic Xe 5p lines. The cluster peak associated with the atomic Xe $5 p_{1 / 2}$ line is considerably narrower than that associate with the Xe $5 p_{3 / 2}$ line. The spectra were found to exhibit considerably more structure than any that have been reported previously, with distinct peaks apparent within the broader bands. Enhanced intensity in the $10.5-11.5 \mathrm{eV}$ binding energy region of the $\mathrm{Xe} 5 p_{3 / 2}$ cluster peak in the spectra measured at 30,40 and $50 \mathrm{eV}$ photon energies was assigned to suppressed contributions from the bulk of the cluster due to the reduced escape depth at these kinetic energies in xenon.

\section{ACKNOWLEDGMENTS}

The Advanced Light Source is supported by the Director, Office of Science, Office of Basic Energy Sciences, of the U.S. Department of Energy under Contract No. DEAC02-05CH11231.

\section{REFERENCES}

1. Hellmut Haberland, Clusters of atoms and molecules : theory, experiment, and clusters of atoms. (SpringerVerlag, Berlin ; New York, 1994).

2. P. M. Dehmer and J. L. Dehmer, Journal of Chemical Physics 68, 3462 (1978).

3. P. M. Dehmer and J. L. Dehmer, Journal of Chemical Physics 69, 125 (1978).

4. A. Wuest and F. Merkt, Journal of Chemical Physics 120, 638 (2004); A. Wuest and F. Merkt, Molecular Physics 103, 1285 (2005).

5. P. Rupper, O. Zehnder, and F. Merkt, Journal of Chemical Physics 121, 8279 (2004).

6. Frank Carnovale, J. Barrie Peel, Richard G. Rothwell et al., Journal of Chemical Physics 90, 1452 (1989).

7. Frank Carnovale, J. Barrie Peel, and Richard G. Rothwell, Journal of Chemical Physics 95, 1473 (1991).

8. R. Feifel, M. Tchaplyguine, G. Oehrwall et al., European Physical Journal D: Atomic, Molecular and Optical Physics 30, 343 (2004).

9. R. Karnbach, M. Joppien, J. Stapelfeldt et al., Review of Scientific Instruments 64, 2838 (1993).

10. N. Berrah, B. Langer, A. A. Wills et al., Journal of Electron Spectroscopy \& Related Phenomena, 101-103, 1, (1999).

11. F. Brandi, I. Velchev, W. Hogervorst et al., Physical Review A 64, 032505 (2001).

12. M. Tchaplyguine, R. R. Marinho, M. Gisselbrecht et al., Journal of Chemical Physics 120, 345 (2004). 